\title{
gs \\ Diffusion-driven superplasticity in ceramics: Modeling and comparison with available data
}

\author{
Diego Gómez-García, ${ }^{1, *}$ Eugenio Zapata-Solvas, ${ }^{1}$ Arturo Domínguez-Rodríguez, ${ }^{1}$ and Ladislas P. Kubin ${ }^{2}$ \\ ${ }^{1}$ Departamento de Física de la Materia Condensada, ICMSE-CSIC, Universidad de Sevilla, Apartado 1065, 41080 Sevilla, Spain \\ ${ }^{2}$ Laboratoire d'Etude des Microstructures, LEM, CNRS-ONERA, 22 Avenue de la Division Leclerc, 92322 Châtillon Cedex, France
}

(Received 15 September 2009; revised manuscript received 3 November 2009; published 8 December 2009)

\begin{abstract}
The discovery of superplasticity in ceramics polycrystals led to debates about whether or not earlier models developed for metallic polycrystals can apply to these systems. In particular, all existing models require some mobility of lattice or grain-boundary dislocations whereas such activity is not observed in most ceramic systems. A model is presented that accounts for the occurrence of superplasticity in the absence of dislocation motion. It is based on a mechanism of grain-boundary sliding by pure-shear motion under stationary conditions, which is accommodated by lattice or grain-boundary diffusion. The prediction of this model regarding the temperature dependences of the stress exponent and of the effective activation energy are found in agreement with experimental results and literature data on five ceramic systems where dislocation activity could not be recorded: $\beta$-SiAlON polycrystals, Al-doped SiC polycrystals, nanocrystalline $\mathrm{MgO}$, yttria-tetragonal zirconia polycrystals, and alumina ceramics polycrystals.
\end{abstract}

DOI: 10.1103/PhysRevB.80.214107

PACS number(s): $62.20 . \mathrm{fq}, 62.20 . \mathrm{Hg}$

\section{INTRODUCTION}

Superplasticity is the ability exhibited by some solids to sustain extremely large plastic elongations without necking, cracking, or being damaged. Typical superplastic elongations can be of several times the initial length of the specimen and much more, in particular, cases. This phenomenon was found in fine-grained polycrystals of pure metals and metallic alloys in the first half of the 20th century. It occurs in specific deformation conditions, typically a temperature higher than half the melting point, a strain rate in the range of conventional laboratory tests $\left(\approx 10^{-4} \mathrm{~s}^{-1}\right)$ within about 2 orders of magnitude and a grain size smaller than $10-20 \mu \mathrm{m}$, which does not evolve during testing. ${ }^{1}$ Superplasticity draws its technological importance from the possibility it offers to form complex components with some very common engineering alloys.

In phenomenological terms, the reason for the occurrence of superplasticity is a high sensitivity of the stress to the strain rate. This property prevents plastic flow from becoming nonuniform by inhibiting the local increase in strain rate associated with the growth of strain localizations. High strain-rate sensitivities can be obtained in polycrystals when plastic flow is controlled by diffusion mechanisms and accommodated by grain-boundary (GB) sliding or vice versa. Although there is general agreement on this point, the underlying mechanisms are still the object of debates..$^{2-4}$

The interest on this phenomenon was renewed by the discovery of superplasticity in ceramics by Wakai ${ }^{5}$ in 1986. Since then, a great effort has been carried out to find new ceramic systems exhibiting a superplastic response at temperatures as low as possible, strain rates as high as possible or both. Despite the obvious technological interest of superplasticity in the field of ceramics, there is a lack of a model specific for this type of materials and having a sufficient predictive ability. Till now, most studies were based on existing models for metallic materials, which involve dislocation activity inside the grains or along the GBs. However, detailed analyses of experimental results show that these models suffer from severe shortcomings when applied to ceramic materials, as illustrated by the careful analysis by Morita and Hiraga ${ }^{6}$ in yttria-tetragonal zirconia polycrystals (Y-TZP). In particular, no dislocation activity at all was detected in many ceramic systems.

Understanding superplasticity in crystalline solids from a fundamental viewpoint is still a challenging task. This is due to the rich diversity of materials and microstructures in which such phenomenon is reported. A nondislocation-based model for superplasticity in ceramics is presented here. It involves very simple assumptions, of which the validity is assessed in several ceramic systems that have been extensively studied, notably Y-TZP. Before deriving this model, several major experimental features of superplasticity and a critical assessment of the current state of the art in modeling must be made.

There is common agreement on the fact that superplasticity is an irreversible process occurring without any microstructural change, this feature being the key to the uniform character of plastic flow. The steady-state strain rate obtained during creep tests on superplastic materials is usually fitted to an equation of the form ${ }^{1}$

$$
\dot{\varepsilon}=A \frac{G b}{k T}\left(\frac{b}{d}\right)^{p}\left(\frac{\sigma}{G}\right)^{n} D,
$$

where $\dot{\varepsilon}$ is the strain rate, $A$ is a constant, $G$ is the shear modulus of the material, $k T$ has its usual meaning, $b$ is a characteristic atomic length of the microstructure, typically the modulus of the Burgers vector, $d$ is the grain size, and $\sigma$ is the constant applied stress. $D$ behaves as a diffusion coefficient and is related to an apparent activation energy $Q$ by an Arrhenius form $D=D_{0} \exp (-Q / k T)$. This form can be experimentally identified as the diffusivity of one or a few of the mobile species in the solid. In such a case, $Q$ truly represents the activation energy of the involved diffusion process. The phenomenological parameters $p$ and $n$ describe the response to changes in grain size and stress; they are com- 
monly called the "grain-size exponent" and the "stress exponent."

A physical theory of superplasticity should be able to account for the following experimental facts: (1) deformation must proceed at constant microstructure. (2) the stress exponent is $n=1$ in many polycrystalline systems but it is also commonly found to be $n=2$ in others. ${ }^{2}$ Furthermore, a transition from $n=1$ to $n=2$ was found upon increasing the flow stress or the grain size.,4 (3) the grain-size exponent $p$ ranges between 2 and 3 in most cases, although the value $p=1$ was occasionally reported. ${ }^{3}$

In what follows, we do not consider models that were developed to explain superplasticity in materials containing a glassy phase (see Meléndez-Martínez et al. ${ }^{7}$ for a review). In such systems, the glassy phase was shown to play a central role as a medium for species solution, migration, and further GB precipitation. As far as fully crystalline materials are concerned, the two most important dislocation-based models that were proposed to explain the experimental data are shortly recalled.

The model by Ball and Hutchinson ${ }^{8}$ states that deformation is due to intergranular dislocation glide accommodated by dislocation climb at GBs. It predicts a parabolic stress dependence of the strain rate and a value $p=2$, irrespective of the flow stress. This model applies to many metallic and nonmetallic systems ${ }^{9}$ but not to all of them. In addition, it cannot explain the transition of the stress exponent $n$ mentioned in the second experimental fact cited above.

The second model, which was proposed by Ashby and Verrall, ${ }^{10}$ involves two sequential processes. It is based on a topological mechanism for GB sliding followed by accommodation by atomic diffusion along the boundaries. This model predicts a linear stress dependence of the strain rate $(n=1)$ and a threshold stress for GB sliding that depends on the interfacial boundary energy. As a consequence, the strain rate should exhibit sigmoidal stress dependence at low stresses, reflecting the presence of the threshold stress. ${ }^{10}$ There are, however, several inconsistencies with experiment. The stress exponent cannot take any other value than $n=1$, the predicted threshold stress values are usually too small to be experimentally detectable and they are much lower than the ones that are sometimes measured. Finally, given the stress, the predicted strain rates are four orders of magnitude smaller than the measured ones.

Artz et al. ${ }^{11}$ further developed an improved version of the original model by Ashby and Verrall. It contains two additions of which only one is significant. It is admitted that grain migration is controlled by the mobility of evenly spaced GB dislocations. The mobility of these dislocations is severely limited by the dragging effect of solutes present in the grain boundaries. Assuming that the dislocation density is proportional to the flow stress, the modified creep equation yields two new features: the stress exponent decreases from $n=2$ at low stresses to $n=1$ with increasing stress while the grain-size exponent increases from $p=1$ to $p=2$.

We emphasize that these models are not to be systematically discarded in the present study. Indeed, the predictions of the Ball-Hutchinson model are verified in many metallic alloys. In fact, this model is likely to apply whenever dislocations glide occurs inside the grains. However, in most non-

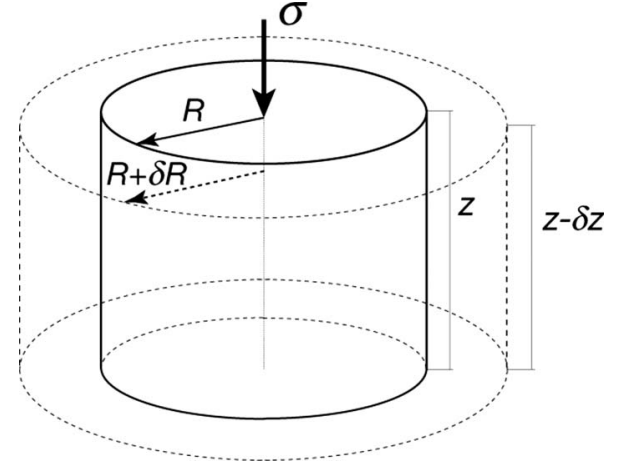

FIG. 1. Macroscopic deformation in compression of a cylindrical sample of radius $R$ and height $z$. In a normal cross section, the motion of the grains is radial.

metallic systems, including ceramics, dislocation activity requires very high applied stresses, which are well above the experimentally recorded ones. In parallel, as already mentioned, postmortem examinations of deformed samples reveal the absence of such dislocation activity. Therefore, there is a lack of understanding for superplasticity in these systems. This constituted the motivation for developing the model that is presented, discussed, and applied to different ceramic systems in the next sections.

\section{MODELING DIFFUSION-DRIVEN SUPERPLASTICITY}

\section{A. Shear events and strain rate.}

We consider a cylindrical polycrystalline specimen with average grain size $d$ submitted to a constant uniaxial compressive stress (Fig. 1). In consistency with the principle of constancy of the microstructure in steady-state conditions, we assume that the temperature is high enough so that the grains can move with respect to each other. As was proposed by Ashby and Verrall, ${ }^{11}$ the microstructure is then periodically altered and reconstructed through the switching of neighboring grains in small clusters. The global result is a heterogeneous deformation along the loading axis produced by the radial shear displacement of some grains in normal cross sections (Fig. 1). We finally assume that grain motion is uncorrelated at times that are large with respect to the time scale of a single event. This was experimentally checked recently in Y-TZP. ${ }^{12}$

The motion of the center of mass of a single grain relative to a contiguous grain can be decomposed as follows. Shear motion, that is a translation parallel to the grain boundary, is mostly responsible for superplastic deformation whereas normal motion is responsible for the so-called accommodation process. The latter is mostly controlled by diffusional mechanisms, specifically lattice or grain-boundary diffusion, and it governs the strain rate. Statistically, a mean flight time, $\delta \tau_{S}$, can be associated to pure-shear motion. This deformation is accommodated through lattice or grain diffusion processes during a mean characteristic time $\delta \tau_{D}$. When accommodation takes place, there is no net additional deformation or it is negligible. In what follows, use is made of the ratio of these two characteristic times, $\beta=\delta \tau_{S} / \delta \tau_{D}$. Figure 2 displays a schematic view of the deformation process. 


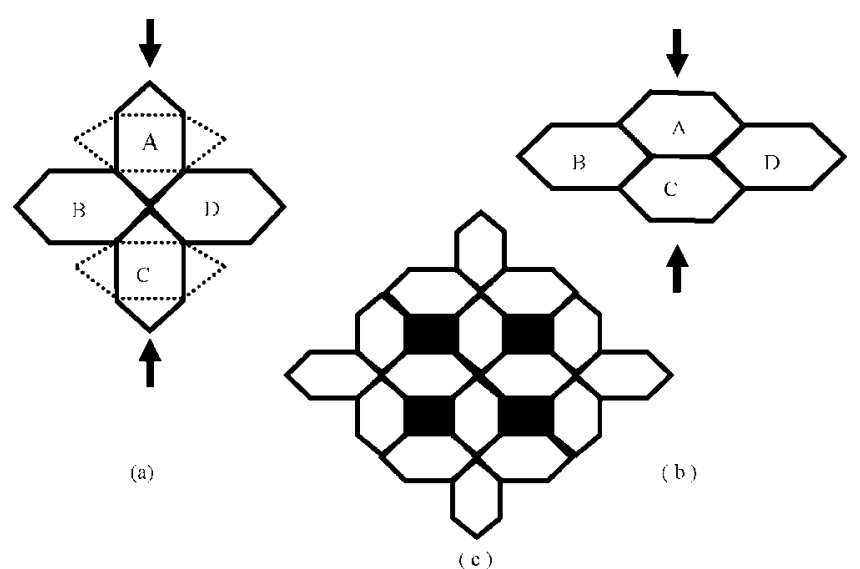

FIG. 2. Scheme showing the elementary process generating deformation. A two-dimensional picture of hexagonal grains is adopted, following the ideas reported by Ashby and Verrall. A set of four grains (a) elongates under compression through rotation (grains $\mathrm{A}$ and $\mathrm{C}$ ), which is in fact a diffusional process, followed by pureshear motion [adopting the configuration (b)]. Rotation is controlled by mass transport during a characteristic time $\delta \tau_{D}$. Pure-shear motion is controlled by voids shrinkage [squares in black in (c)] during a typical time $\delta \tau_{S}$. This motion is ruled by the curvature change in the interfaces in contact. It is important to emphasize that shear motion demands the existence of voids which must be created by grain growth or shrinkage of adjacent grains.

The condition of volume conservation during plastic deformation is written as $z d S+S d z=0$, where $S$ is the crosssectional area and $z$ is the height of the sample (Fig. 1). In compression, the strain rate is given by

$$
\dot{\varepsilon}=\frac{d \dot{z}}{z}=-\frac{d \dot{S}}{S},
$$

where the dots denote derivation with respect to time.

During steady-state deformation, and in the absence of statistical time correlations, the grains move stochastically by a Markov process. ${ }^{13}$ In such conditions, the net deformation is produced by a constant number of grains $N$. The number of grains moving by pure-shear events per unit time, $\dot{N}_{\text {shear }}$ is then

$$
\dot{N}_{\text {shear }}=W_{0 \rightarrow 1} \frac{N}{\delta \tau_{S}}-W_{1 \rightarrow 0} \frac{N}{\delta \tau_{D}},
$$

where $W_{0 \rightarrow 1}$ is the probability for a transition from predominant normal motion (state " 0 ") to a pure-shear displacement (state " $1 ")$ and $W_{1 \rightarrow 0}$ is the probability for the reverse transition. In steady state, $\dot{N}_{\text {shear }}=0$, hence $N$ is a constant. With $W_{0 \rightarrow 1}+W_{1 \rightarrow 0}=1$, one obtains

$$
W_{0 \rightarrow 1}=\frac{\delta \tau_{S}}{\delta \tau_{S}+\delta \tau_{D}}=\frac{\beta}{1+\beta} .
$$

This type of relation is typical of queuing theories. ${ }^{14}$

During pure-shear motion, the center of a grain has a velocity $\alpha d / \delta \tau_{S}$, where $\alpha$ is a numerical factor. One necessarily has $\alpha<1$ because shear motion is associated to accommodation steps. The parameter $\alpha$ was evaluated by
Padmanabhan ${ }^{15}$ in the way suggested by Gifkins and Langdon, ${ }^{2}$ taking into account that the nonspherical character of the grains limits grain-boundary sliding. For twodimensional hexagonal grains the value found is $\alpha \approx 0.07$. In general, $\alpha$ depends on grain topology. In materials with almost equiaxed grains, $\alpha \approx 0.5$, that is, $\alpha d$ is the radius of the sphere circumscribing an average grain.

The whole shearing and accommodation processes occur during a time interval $\delta \tau=\delta \tau_{S}+\delta \tau_{D}$, which is the sum of the characteristic times for pure shear and pure accommodation. Denoting by $\delta \bar{w}$ the average radial displacement per grain that results from a shearing event, the mean displacement rate per grain, $\delta \bar{w} / \delta \tau$, is written as

$$
\frac{\delta \bar{w}}{\delta \tau}=\frac{\alpha d}{\delta \tau_{S}} \frac{\delta \tau_{S}}{\delta \tau_{S}+\delta \tau_{D}}=\frac{\alpha d}{\delta \tau_{D}(1+\beta)} .
$$

During the time interval $\delta \tau$, the area of a normal cross section increases at a rate $\dot{S}=2 \pi R \dot{R}$, where $R$ is the radius of the sample (Fig. 1). In steady state, the rate at which the radius $R$ increases is the product of the constant number of shearing events, $N_{\text {shear }}$, by the average displacement rate per grains as given by Eq. (5). The increment of section is then given by

$$
d \dot{S}=2 \pi R N_{\text {shear }} \frac{\alpha d}{\delta \tau_{D}(1+\beta)} .
$$

The number of pure-shear events in steady-state regime, $N_{\text {shear }}$, is the product of the number of grains along the radial direction $(R / d)$ by the probability for a shear motion, $W_{0 \rightarrow 1}$, which is given by Eq. (4). In conditions of constant volume [Eq. (2)], the strain rate is eventually written as

$$
\dot{\varepsilon}=\frac{d \dot{S}}{S}=\frac{2 \alpha \beta}{(1+\beta)^{2}} \frac{1}{\delta \tau_{D}}=\frac{2 \alpha \delta \tau_{S}}{\left(\delta \tau_{S}+\delta \tau_{D}\right)^{2}} .
$$

\section{B. Superplastic strain rate}

To express the strain rate in terms of stress and temperature, the characteristic times $\delta \tau_{S}$ and $\delta \tau_{D}$ have to be estimated. The characteristic time for diffusion is straightforwardly obtained from diffusion theory. Taking a diffusion path of length $\alpha d$ and a driving force deriving from the gradient of chemical potential on the grains, one obtains ${ }^{16}$

$$
\delta \tau_{D}=\frac{\alpha d}{\sigma \Omega D_{\mathrm{eff}} / k T \alpha d}=\frac{\alpha^{2} d^{2}}{\sigma \Omega D_{\mathrm{eff}}} k T
$$

where $\sigma$ is the applied stress, $\Omega$ is the atomic volume of the diffusing species controlling accommodation, and $D_{\text {eff }}$ is the effective diffusion coefficient along the possible diffusion paths. It is worth noting that this diffusion coefficient is a linear combination of the lattice and the grain-boundary diffusion coefficients, respectively, $D_{\ell}$ and $D_{\mathrm{gb}}$ (Ref. 16): $D_{\mathrm{eff}}$ $=D_{\ell}+\pi \delta D_{\mathrm{gb}} / d$. In this expression, $\delta$ is the thickness of the grain-boundary diffusion layer, which is assimilated to the lattice parameter. Depending upon the value of $\pi \delta / d$, the effective diffusion process can be approximated by lattice or grain diffusion processes. Thus, the controlling diffusion mechanism can usually be determined from experimental values of the activation energy. 
Combining Eqs. (7) and (8), one obtains

$$
\dot{\varepsilon}=\frac{2 \beta}{(1+\beta)^{2}} \frac{\sigma \Omega}{k T \alpha d^{2}} D_{\text {eff }} .
$$

To determine the ratio $\beta=\delta \tau_{S} / \delta \tau_{D}$, one has to know the temperature and stress dependences of $\delta \tau_{S}$. This last quantity depends on the system under study through the mechanism that governs grain-boundary mobility during a shearing event.

In some systems, grain-boundary motion is controlled by the Herring equation, ${ }^{17}$ which expresses that the motion of a curved grain boundary is driven by the gradient of its surface energy. In other words, the motion is driven by the tendency of grain surfaces to flatten under the effect of their interfacial energy $\gamma$. In a linear approximation, the velocity of a grain, $d x / d t$, is proportional to its mean curvature $2 / x$, where $x$ is the normal distance from the grain boundary to the center of curvature of the grain. One then has

$$
\frac{d x}{d t}=\mu \frac{2 \gamma}{x}
$$

where $\mu$ is the grain-boundary mobility. Thus, for a shear displacement $\alpha d$, Eq. (11) is integrated in the form

$$
\delta \tau_{S}=\int_{d / 2}^{(1+\alpha / 2) d / 2} \frac{x d x}{2 \gamma \mu}=\frac{\alpha\left(1+\alpha^{2} / 4\right) d^{2}}{16 \gamma \mu} .
$$

The choice of the upper integration limit is consistent with the Ashby-Verrall's model. ${ }^{10}$ The total displacement is $\alpha d$ and pure-shear motion requires a grain curvature equal to one half of the displacement induced by pure diffusion motion (cf. Fig. 4 of Ref. 10). This pure diffusion motion produces a strain of 0.55 , in good agreement with the choice of $\alpha d / 4$ for the change in curvature value. Combining Eqs. (8) and (11), one obtains an expression for $\beta$,

$$
\beta=\frac{\sigma \Omega\left(1+\alpha^{2} / 4\right)}{16 \alpha k T} \frac{D_{\mathrm{eff}}}{\mu \gamma} .
$$

The grain mobility is proportional to the grain-boundary diffusivity, $D_{\mathrm{gb}}$, through a relation that involves the thickness of the grain-boundary layer, ${ }^{18}$

$$
\mu=\frac{\Omega}{2 \pi k T \delta} D_{\mathrm{gb}} .
$$

Thus, in the considered type of systems, one finally obtains

$$
\beta=\frac{\pi\left(1+\alpha^{2} / 4\right)}{8 \alpha} \frac{\sigma \delta}{\gamma} \frac{D_{\mathrm{eff}}}{D_{\mathrm{gb}}} .
$$

In other systems, particularly in the ones where grainboundary mobility is limited by solute drag, grain-boundary motion usually follows the relation: ${ }^{17}$

$$
\frac{d x}{d t}=\mu \frac{2 \gamma \delta}{x^{2}} .
$$

The characteristic time associated with a shearing event, is obtained by integration, as was done above. One straightforwardly obtains

$$
\delta \tau_{S}=\frac{\alpha d^{3}}{32 \gamma \delta \mu}\left(1+\frac{\alpha}{2}+\frac{\alpha^{2}}{12}\right) .
$$

The grain mobility has same expression as in Eq. (13) with, however, a different mechanism associated with the diffusivity $D_{\mathrm{gb}}$. Combining Eqs. (8), (13), and (16), one obtains another expression for $\beta$ as a function of stress, grain size, and temperature,

$$
\beta=\frac{\pi}{16} \frac{\sigma d}{\gamma \alpha}\left(1+\frac{\alpha}{2}+\frac{\alpha^{2}}{12}\right) \frac{D_{\mathrm{eff}}}{D_{\mathrm{gb}}} .
$$

An important aspect must be emphasized before continuing with the implications of this model. Equations (10) and (15) assume that shear motion is essentially driven by the curvature effects of the grain boundaries. Such assumption is a key ingredient of this model, which had not been considered previously. It is consistent with the recent numerical models. ${ }^{19}$ The curvature effects have been shown to play a remarkable role on the segregation of aliovalent cations to the boundaries in some superplastic ceramic systems. ${ }^{20,21}$

\section{Limiting cases}

Two limiting cases, which correspond to the most usual experimental conditions, will be considered in what follows. In the first limiting case, the shearing step is much faster than the accommodation step and one has $\beta \ll 1$. This case is typically met in low-stress high-temperature conditions, as further discussed in Sec. II D. Then, Eq. (9) can be approximated by

$$
\dot{\varepsilon} \approx \frac{2 \beta}{\alpha d^{2}} \frac{\sigma \Omega}{k T} D_{\text {eff }} .
$$

When the shearing velocity of grains is governed by curvature, $\beta$ is given by Eq. (14). The creep rate given by Eq. (18) can be expressed in the form

$$
\dot{\varepsilon}=A \frac{G b}{k T}\left(\frac{\sigma}{G}\right)^{2}\left(\frac{b}{d}\right)^{2} D^{\prime},
$$

where $A=A_{\mathrm{o}} \delta G \Omega / \gamma b^{3}$, with $A_{\mathrm{o}}=\left(1+\alpha^{2} / 4\right) \pi / 8 \alpha^{2}$, is a dimensionless constant and $D^{\prime}=D_{\mathrm{eff}}^{2} / D_{\mathrm{gb}}$ has the dimension of a diffusion coefficient.

When the shearing rate is governed by solute drag, $\beta$ is given by Eq. (17) and the creep equation is written as

$$
\dot{\varepsilon}=A^{\prime} \frac{G b}{k T}\left(\frac{\sigma}{G}\right)^{2}\left(\frac{b}{d}\right) D^{\prime} .
$$

The value of the dimensionless constant is now $A^{\prime}$ $=A_{\mathrm{o}}^{\prime} G \Omega / \gamma b^{2}$, with $A_{\mathrm{o}}^{\prime}=\pi\left(1+\alpha / 2+\alpha^{2} / 12\right) / 16 \alpha^{2} \gamma b^{2}$.

In this limiting high-temperature case, non-Newtonian flow is obtained from the condition $\beta \ll 1$, which implies large shearing velocities. In such conditions, grain growth may be expected to prevent the occurrence of a large-scale superplastic regime. This is, indeed, observed in some ceramic systems, in which extensive grain growth is associated to plastic flow, microstructural evolution, and strain hardening. Nevertheless, superplastic behavior is obtained in many other ceramic materials, notably yttria-tetragonal zirconia 
polycrystals, before the onset of appreciable grain growth. The analysis of experimental results presented in Sec. III D is exclusively dealing with such ceramic systems.

In the second limiting case, the two characteristic times are similar and $\beta \approx 1$. The creep rate, as given by Eq. (9), is then written as

$$
\dot{\varepsilon}=B \frac{G b}{k T}\left(\frac{\sigma}{G}\right)\left(\frac{b}{d}\right)^{2} D_{\text {eff }} .
$$

Equation (21) has same functional form as the one proposed by Herring ${ }^{19}$ except for the value of the constant, which is here $B=\Omega / 2 \alpha b^{3}$. However, in contrast to what is assumed in the model by Nabarro and Herring, ${ }^{17}$ plastic flow is still governed by grain-boundary sliding.

The case where $\beta>1$ is not considered here. It corresponds to a deformation process with microstructural evolution so that no superplastic behavior can be obtained.

\section{Phenomenological stress and temperature dependences}

Stress dependence. The superplastic behavior is usually fitted to a power law. The stress exponent $n$, which is defined in Eq. (1), is $n=2$ in Eqs. (20) and (21), that is when $\beta=0$ whereas it is $n=1$ in Eq. (22) when $\beta=1$. One can notice that the values of $\beta$ given by Eqs. (14) and (17) are proportional to the stress $\sigma$. This allows determining an effective stress exponent, $n_{\mathrm{eff}}$, from Eq. (9),

$$
n_{\mathrm{eff}}=\left(\frac{\partial \ln \dot{\varepsilon}}{\partial \ln \sigma}\right)_{T}=\frac{2}{1+\beta} .
$$

Thus, the present model predicts that during superplastic flow the stress exponent decreases with increasing stress and varies continuously from $n=2$ to $n=1$.

Temperature dependence of the strain rate. The temperature dependence is mostly fitted to an Arrhenius form, with an activation energy $Q$ given by $Q=k T^{2}(\partial \ln \dot{\varepsilon} / \partial T)_{\sigma}$. The partial derivative is calculated from Eq. (9); in this equation, the ratio $\beta$ of the characteristic times for diffusion and shear is of the form $\beta \propto \exp \left[-\left(Q_{\text {eff }}-Q_{\mathrm{gb}}\right) / k T\right]$, where $Q_{\text {eff }}$ is the effective activation energy for diffusion and $Q_{\mathrm{gb}}$ is the activation energy for grain-boundary diffusion. Neglecting a small term $k T$, one eventually obtains

$$
Q=Q_{\mathrm{eff}}+\frac{1-\beta}{1+\beta}\left(Q_{\mathrm{eff}}-Q_{\mathrm{gb}}\right) .
$$

One can see that $Q \approx 2 Q_{\mathrm{eff}}-Q_{\mathrm{gb}}$ when $\beta \ll 1$ and $Q \approx Q_{\text {eff }}$ when $\beta \approx 1$. In most cases, $Q_{\text {eff }}$ is very close to the activation energy for lattice diffusion, particularly if the grain size is large enough.

It is important to emphasize that these two dependences cannot be explained in the frame of a dislocation-based model. Such model would predict a nontemperature dependence of the activation energy. Moreover, it would predict a constant value of the stress exponent $(n=2)$ in the whole range of stress and temperatures ${ }^{8}$ of plastic regime or only for very small stresses if the modified version ${ }^{11}$ is considered. Such predictions are just the opposite of those derived from the model discussed in this paper. We will see in the next section that experimental observations are consistent with this model and cannot be understood in the context of a dislocation-driven model.

\section{EXPERIMENTAL VALIDATION}

Most superplastic ceramic materials display either a linear stress dependence or a parabolic one. A linear dependence is consistent with grain-boundary sliding accommodated by diffusion. ${ }^{10}$ In contrast, the parabolic dependence was, sometimes, implicitly discussed in terms of the Ball-Hutchinson's model, ${ }^{8}$ even though no dislocation activity was observed. Thus, it actually remains unexplained. Five different ceramic systems are discussed in this section and their mechanical behavior is compared to the expectations of the present model.

\section{A. $\beta$-SiAlON polycrystals}

Chihara et al. $^{22}$ have recently investigated the hightemperature compressive deformation of $\beta$-SiAlON polycrystals. The temperatures considered ranged between 1823 and $1973 \mathrm{~K}$. The authors reported a stress exponent of $n$ $\approx 2$, which decreases toward $n \approx 1$ when the flow stresses increases. It was concluded that dislocations played no important role in the deformation of this material, in spite of the suggestion that the Artz-Ashby-Verrall model ${ }^{11}$ may account for the observed transition in stress-exponent values.

The activation energy was found to depend on stress (or temperature). Its value decreases from $Q_{1} \approx 1200 \mathrm{~kJ} / \mathrm{mol}$ for the lowest stresses $(\beta \ll 1)$ to $Q_{2} \approx 900 \mathrm{~kJ} / \mathrm{mol}$ for the highest ones $(\beta \approx 1)$. Such dependence is consistent with the prediction of Eqs. (22) and (23). One should then have $Q_{\mathrm{eff}}$ $\approx 900 \mathrm{~kJ} / \mathrm{mol}$ and $Q_{\mathrm{gb}} \approx 600 \mathrm{~kJ} / \mathrm{mol}$. In Ref. 20 the flow stress values for which the stress exponent is $n \approx 1.5$ were also determined as a function of temperature. From Eq. (22), a value $n \approx 1.5$ corresponds to $\beta \approx 0.3$ and one deduces from Eq. (14) or (17) that the temperature dependence of the flow stress $\sigma_{1.5}$ should follow an Arrhenius relation of the form

$$
\sigma_{1.5} \propto \exp \left(\frac{Q_{\mathrm{eff}}-Q_{\mathrm{gb}}}{k T}\right) .
$$

An Arrhenius plot of the experimental $\sigma_{1.5}$ values drawn from Ref. 22 is shown in Fig. 2. Although the experimental accuracy of these measurements is not known, the agreement with Eq. (24) looks quite reasonable, all the more as the experimental slope yields an activation energy of $300 \mathrm{~kJ} /$ mol, which is equal to $\left(Q_{\mathrm{eff}}-Q_{\mathrm{gb}}\right)$.

\section{B. Al-doped SiC polycrystals}

Tokiyama et $a .^{23}$ have recently studied the hightemperature deformation of Al-doped SiC polycrystals. The authors report a transition in the stress-exponent value, which increases from $n \approx 1.4$ to $n \approx 2.6$ when the stress decreases. These measurements were performed at a single temperature $\left(1850{ }^{\circ} \mathrm{C}\right.$ ) and no information is available on the activation energies. No evidence of dislocation activity was reported. This seems reasonable because the Frank-Read 
stress is expected to be much larger than the flow stress in the range of experimental conditions investigated. The model by Artz et $a l .{ }^{11}$ is also invoked in that case to explain the transition in stress-exponent values, despite the absence of dislocation activity.

The reason for the occurrence of a stress exponent $n$ $\approx 2.6$ is discussed as follows. ${ }^{23}$ The model by Artz, Ashby, and Verrall states that the flow stress is proportional to the number of grain-boundary dislocations. When the number $N$ of dislocation per grain-boundary interface is limited, a multiplicative factor $N^{2} /\left(N^{2}+1 / 2\right)$ is introduced in the conventional equation for steady-state creep. Therefore, when $N$ is vanishingly small, this factor can be approximated by $2 N^{2}$ and the strain rate should depend on the flow stress cubed $\left(2 N^{2} \times N\right)$. This statement is, however, incorrect because $N$ cannot take values between 0 and 1 . Furthermore, the transition in stress-exponent values is reported to occur at a flow stress of about $250 \mathrm{MPa}$ (the stress is in the range of 30-400 $\mathrm{MPa}$ ). This transition is smooth whereas it should be very sharp if it were to be explained by the multiplicative factor; $N$ should then increase by more than 1 order of magnitude in the considered stress range and the multiplicative factor should converge to 1 very fast.

The present model qualitatively accounts for the transition and an estimate of the transition stress can be performed. The lattice parameter of silicon carbide ${ }^{24}$ is $a \approx 3.1 \AA$ and the grain-boundary energy per unit area ${ }^{25}$ is $\gamma \approx 0.1-0.3 \mathrm{~J} \mathrm{~m}^{-2}$. Like in Sec. III A, we take the transition stress at $n=1.5$, which corresponds to $\beta=0.3$. Tokiyama et al. ${ }^{23}$ indicate that accommodation is mainly driven by grain-boundary diffusion, thus $D_{\mathrm{eff}} \approx D_{\mathrm{gb}}$. With $\delta \approx a$ and $\alpha \approx 0.5$, Eq. (14) yields a transition stress between 116 and $348 \mathrm{MPa}$, which is of the right order of magnitude when compared to the experimental value of $250 \mathrm{MPa}$. The value $n=2.6>2$ might be associated to the existence of a small threshold stress for grainboundary sliding, as was previously suggested by Ashby and Verrall. ${ }^{10}$

\section{Nanocrystalline MgO}

Fully dense nanocrystalline $\mathrm{MgO}$ specimens were tested in compression at $750{ }^{\circ} \mathrm{C}$ by Domínguez-Rodríguez et al. ${ }^{26}$ The average grain size was $30-50 \mathrm{~nm}$. In such conditions, no dislocation activity was detected, extensive grain-boundary sliding was observed and the stress exponent was $n=2$ all through the range of investigated flow stresses. Thus, none of the classical models for superplasticity applies to this system. The present model is compatible with these results, taking into account the high values of the grain-boundary energies in $\mathrm{MgO}$ reported by Keliman and Chaim. ${ }^{27}$ To estimate the value of $\beta$, we relate the effective diffusion coefficient to the grain-boundary diffusion coefficient by $D_{\text {eff }} \approx \pi \delta D_{\mathrm{gb}} / d$ (Ref. 26, see also Sec. II B). With the typical values $\sigma$ $\approx 300 \mathrm{MPa}, \gamma \approx 1.1 \mathrm{~J} \mathrm{~m}^{-2}, \alpha \approx 0.5$, and $\delta \approx 4 \AA$, Eq. (14) yields $\beta \approx 3 \times 10^{-3}$. It follows from Eq. (22) that $n \approx 2$, which is precisely the experimentally obtained value.

\section{Yttria-tetragonal zirconia polycrystals}

Y-TZPs are one of the most studied structural ceramics system and their high-temperature mechanical behavior is

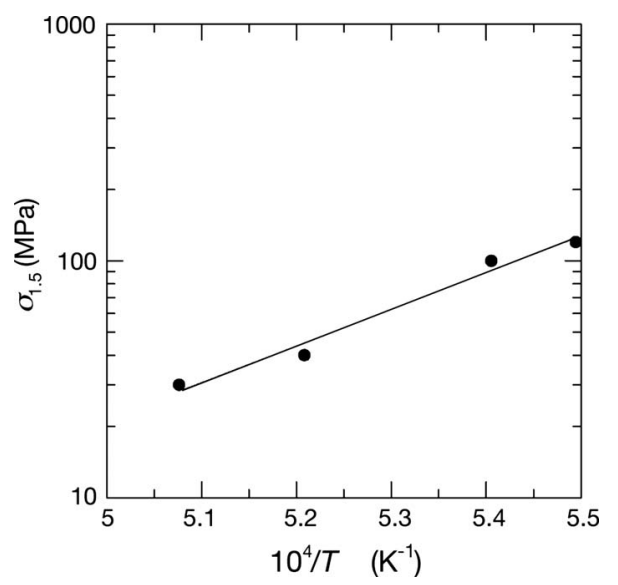

FIG. 3. Temperature dependence of the flow stress $\sigma_{1.5}$, corresponding to a stress exponent $n=1.5$, in $\beta$-SiAlON polycrystals (redrawn from Chihara et al. (Ref. 22); (no error bars are provided in this reference). The agreement between the experimental data and the Arrhenius form predicted by Eq. (24) is quite good. The straight line corresponds to the expected activation energy of 300 $\mathrm{kJ} / \mathrm{mol}$ (see text).

extensively described in the literature (cf. the review by Jiménez-Melendo et $a .^{28}$ ). It is widely accepted that grainboundary sliding is the dominant process responsible for large deformations and the stress exponent is found to be $n$ $=2$ in the superplastic regime. The typical grain size of superplastic YTZP specimens is $0.2-0.5 \mu \mathrm{m}$. Recently, Zapata-Solvas et al. ${ }^{29}$ measured the dependence of the stress exponent on grain size at constant strain rate and found that it decreases with increasing grain size. In parallel, the grainsize exponent is constant $(p=2)$. A few years ago, the nature of the deformation mechanism in this system was the subject of a controversy. Whereas Berbon and Langdon ${ }^{30}$ considered that the Artz-Ashby-Verrall mechanism ${ }^{11}$ applies in that case, Domínguez-Rodríguez and Jiménez-Melendo ${ }^{31}$ and Morita and Hiraga ${ }^{6}$ opposed this view. At present, none of the current models can account for the values of the stress exponent.

Under a constant strain rate, the larger the grain size, the larger the flow stress according to Eq. (1) taken with $n \leq 2$ and $p=2$. Thus, increasing the grain size implies increasing the value of $\beta$. From Eqs. (14) and (17), $\beta$ is proportional to stress and grain size. Inserting this result in Eq. (9), one finds after some manipulation that $\sigma /(\dot{\varepsilon} d)^{1 / 2}$ should be proportional to $\sigma d$ at a given temperature. Zapata-Solvas et al. ${ }^{29}$ measured the stress dependence of the strain rate as a function of stress at $1350{ }^{\circ} \mathrm{C}$ for Y-TZP specimens with different grain sizes. Figure 3 shows a plot of $\sigma /(\dot{\varepsilon} d)^{1 / 2}$ as a function of $\sigma d$, which combines the data obtained in Ref. 29 with another set of data ${ }^{28}$ at the same temperature. A good linear fit is obtained in spite of the experimental scatter inherent to high-temperature measurements.

\section{E. Polycrystalline alumina ceramics}

Finally, several alumina ceramics exhibit the same behavior as Y-TZP. The analysis performed in this section is applied to this case and illustrated by Fig. 4. This plot is similar 


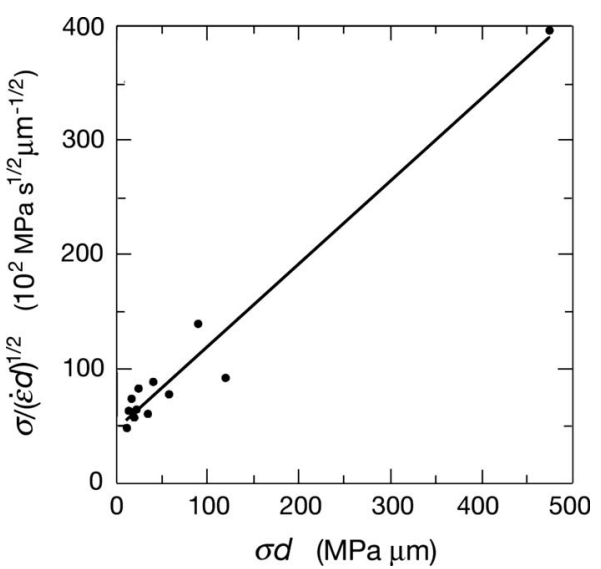

FIG. 4. Plot of $\sigma /(\dot{\varepsilon} d)^{1 / 2}$ versus $\sigma d$ constructed from results on Y-TZP ceramics at $1350{ }^{\circ} \mathrm{C}$ by Melendo et al. (Ref. 28) and Zapata-Solvas et al. (Ref. 29). The present model predicts that all data should follow a linear dependence in such a plot. The regression line fits the data with a regression coefficient $r=0.996$. The data values are estimated to be within $\pm 10 \%$, which is typical for high-temperature measurements. The inaccuracies result from the measurement of stresses $( \pm 2 \%)$, strain rates $( \pm 10 \%)$, and grain sizes $( \pm 5 \%)$.

to the one of Fig. 3. The data are drawn from literature on alumina ceramics deformed at $1300{ }^{\circ} \mathrm{C}$ (Refs. 32-34) and the results are again found in fairly good agreement with the predicted dependence (Fig. 5).

\section{CONCLUSIONS}

A model was developed for explaining the hightemperature superplastic properties of ceramic systems. This model is established on very simple physical bases. A key element of this model is the central role of grain curvature as

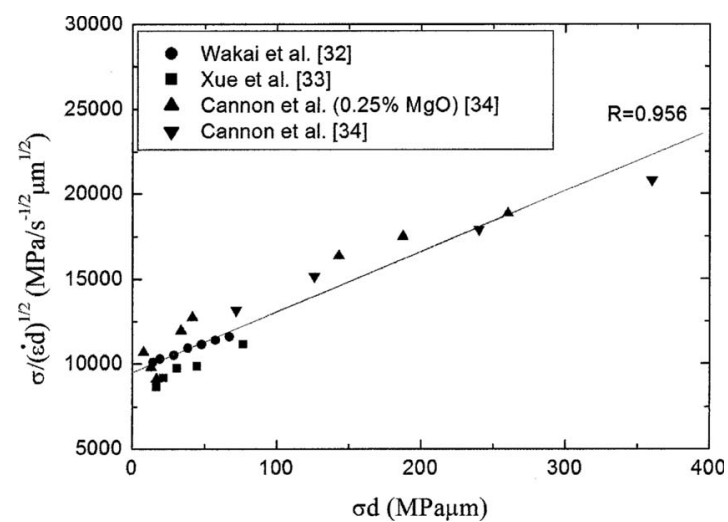

FIG. 5. Plot of $\sigma /(\dot{\varepsilon} d)^{1 / 2}$ versus $\sigma d$ at $1300{ }^{\circ} \mathrm{C}$ for several alumina ceramics. The data are taken from published results (Refs. 32-34) and include alumina (Ref. 34) doped with $\mathrm{MgO}$. The regression line is obtained with a regression factor $r=0.956$. The sources of experimental error are the same as in Fig. 3 and the data values are also estimated to be typically within $\pm 10 \%$.

the driving force for grain sliding. Assuming that grain boundaries are dislocation free, it describes a mechanism of grain-boundary sliding accommodated by diffusion, which can explain the values of the stress exponent, especially the value $n=2$, and its possible stress and temperature dependences. The consequences are found to be consistent with most experimental results in ceramic systems where no dislocation activity could be detected.

\section{ACKNOWLEDGMENTS}

The authors gratefully acknowledge O. A. Ruano (Centro Nacional de Investigaciones Metalúrgicas, Madrid, Spain) for many fruitful discussions. This work was supported by the Funds awarded by the Spanish "Ministerio de Ciencia e Innovación" (Grants No. MAT2006-10249-C02-02 and No. MAT2009-14351-C 02-01).
*Corresponding author. FAX: +954612097; dgomez@us.es

${ }^{1}$ J. P. Poirier, Creep in Crystals (Cambridge University Press, Cambridge, United Kingdom, 1985).

${ }^{2}$ R. C. Gifkins and T. G. Langdon, Mater. Sci. Eng. 36, 27 (1978).

${ }^{3}$ R. C. Gifkins and T. G. Langdon, Mater. Sci. Eng. 40, 293 (1979).

${ }^{4}$ K. A. Padmanabhan, Mater. Sci. Eng. 40, 285 (1979).

${ }^{5}$ F. Wakai, S. Sakaguchi, and Y. Matsuno, Adv. Ceram. Mater. 1 (3), 259 (1986).

${ }^{6}$ K. Morita and K. Hiraga, Scr. Mater. 48, 1403 (2003).

${ }^{7}$ J. J. Meléndez-Martínez, D. Gómez-García, and A. DomínguezRodríguez, Philos. Mag. 84, 2305 (2004).

${ }^{8}$ A. Ball and M. M. Hutchinson, Metal. Sci. J 3, 1 (1969).

${ }^{9}$ O. A. Ruano, J. Wadsworth, and O. D. Sherby, Acta Mater. 51, 3617 (2003).

${ }^{10}$ M. F. Ashby and R. A. Verrall, Acta Metall. 21, 149 (1973).

${ }^{11}$ E. Arzt, M. F. Ashby, and R. A. Verrall, Acta Metall. 31, 1977 (1983).
${ }^{12}$ K. Yasuda, T. Okamoto, T. Shiota, and Y. Matsuo, Mater. Sci. Eng., A 418, 115 (2006).

${ }^{13}$ N. G. Van Kampen, Stochastic Processes in Physics and Chemistry (Elsevier Science, The Netherlands/North-Holland, Amsterdam, 1990).

${ }^{14}$ B. P. Roe, Probability and Statistics in Experimental Physics. (Springer-Verlag, Berlin, Germany, 2001).

${ }^{15}$ K. A. Padmanabhan, Mater. Sci. Eng. 29, 1 (1977).

${ }^{16}$ J. Philibert, Atom Movements, Diffusion and Mass Transport in Solids (EDP Sciences, Paris, France, 1981).

${ }^{17}$ A. Michels, C. E. Krill, H. Ehrhardt, R. Birringer, and D. T. Wu, Acta Mater. 47, 2143 (1999).

${ }^{18}$ W. D. Kingery, Introduction to Ceramics, Series on Science and Technology of Materials, 2nd ed. (Wiley, New York, USA, 1995).

${ }^{19}$ F. Wakai, Y. Shinoda, S. Ishihara, and A. Domínguez-Rodríguez, Acta Mater. 50, 1177 (2002).

${ }^{20}$ D. Gómez-García, C. Lorenzo-Martín, A. Muñoz-Bernabé, and A. Domínguez-Rodríguez, Phys. Rev. B 67, 144101 (2003). 
${ }^{21}$ J. W. Cahn, Y. Mishin, and A. Suzuki, Acta Mater. 54, 4953 (2006).

${ }^{22}$ K. Chihara, D. Hiratsuka, Y. Shinoda, T. Akatsu, F. Wakai, J. Tatami, and K. Komeya, Mater. Sci. Eng., B 148, 203 (2008).

${ }^{23}$ T. Tokiyama, Y. Shinoda, T. Akatsu, and F. Wakai, Mater. Sci. Eng., B 148, 261 (2008).

${ }^{24}$ A. Bauer, J. Kräußlich, L. Dressler, P. Kuschnerus, J. Wolf, K. Goetz, P. Kräckell, J. Furthmüller, and F. Bechstedt, Phys. Rev. B 57, 2647 (1998).

${ }^{25}$ T. Takai, T. Halicioglu, and W. A. Tiller, Surf. Sci. 164, 341 (1985).

${ }^{26}$ A. Domínguez-Rodríguez, D. Gómez-García, E. Zapata-Solvas, and R. Chaim, Scr. Mater. 56, 89 (2007).
${ }^{27}$ S. Kleiman and R. Chaim, Mater. Lett. 61, 4489 (2007).

${ }^{28}$ M. Jiménez-Melendo, A. Domínguez-Rodríguez, and A. BravoLeón, J. Am. Ceram. Soc. 81 [11], 2761 (1998).

${ }^{29}$ E. Zapata-Solvas, D. Gómez-García, C. García-Gañán, and A. Domínguez-Rodríguez, J. Eur. Ceram. Soc. 27, 3325 (2007).

${ }^{30}$ M. Z. Berbon and T. G. Langdon, Acta Mater. 47, 2485 (1999).

${ }^{31}$ M. Jiménez-Melendo and A. Domínguez-Rodríguez, Acta Mater. 48, 3201 (2000).

${ }^{32}$ F. Wakai, T. Iga, and T. J. Nagano, J. Ceram. Soc. Jpn. 96, 1206 (1988).

${ }^{33}$ L. A. Xue and I. W. Chen, J. Am. Ceram. Soc. 73, 3518 (1990).

${ }^{34}$ R. M. Cannon, W. H. Rhodes, and A. H. Heuer, J. Am. Ceram. Soc. 63, 46 (1980). 